\title{
THE RELEVANCE OF INFORMATION SYSTEMS IN ORGANISATIONS: A REVIEW OF EXISTING LITERATURE
}

\author{
Forgive Kwadamah ${ }^{1}$ and Francis Pol Lim ${ }^{2}$ \\ ${ }^{1}$ College of Distance Education, Zenith University College Centre, \\ University of Cape Coast, Ghana \\ ${ }^{2}$ SMC University, Zug, Switzerland \\ forgivekwadamah@yahoo.com ${ }^{1}$, limfrancispol19@gmail.com ${ }^{2}$
}

\begin{abstract}
This paper reviews monumental issues pertaining to technology and its usage in the management of information in organisations. The paper recognizes the remarkable progress the world has made through technology. It touches on the novelty introduced by technology in the operations of individual organisations in particular and the society in general. It focuses on the key issues that arose in the early days of integrating technology with information management in organisations and the essence of management information systems in modern-day organisations. It also addresses the perceptions regarding information systems and businesses dimensions of information systems in organisations - the organisation, its management and technology. The paper vividly dissects the two main computer networks, intranet and extranet, used in organizations and their usefulness in business entities. The paper finally touches on the four major types of information systems normally found in business organisations and their relevance in business organisations.
\end{abstract}

Keywords - Information Systems, Transaction Processing Systems (TPS), Management Information Systems (MIS), Decision Support Systems (DSS), Executive Support Systems (ESS)

\section{INTRODUCTION}

Information technology (IT) has permeated every fabric of life. It has revolutionised all dimensions of human society: the smallest community to the global community (WenLung, 2015). Sophisticated technology has touched and improved the lives of mankind incredibly. Higher technological services, in the form of open source software and cloud computing, have introduced novel prospects in the information technology (IT) industry and has undoubtedly impacted the existence of humankind (Wen-Lung, 2015). Management information systems stated receiving recognition for almost four decades. From the early 1980s, the Society for Information Management (SIM) has assessed consultants and academics in IT and Information System disciplines in order to comprehend the most critical challenges in the discipline, rating of such challenges, and spell out arrangements concerning challenges/rating among participants (Dickson, Leitheiser, Weitherbe \& Nechis (1984). This intermittent review has become fundamental to the progress of management information systems (MIS). The results of the seasonal probe has turned out to be a momentous point of reference for MIS scholars ubiquitously and conferred with when deliberations are underway on investigations in the discipline (Wen-Lung, 2015). 
The heavy dependency of the whole world on technology is becoming progressively phenomenal. Therefore, crucial MIS matters have also turned out to be more important when business entities are making the effort to invest in IT for corporate benefits (WenLung, 2015). Managers need to be aware of the economies of scale associated with the introduction of MIS in their organisations in addition to its attendant challenges.

\section{KEY ISSUES IN MIS}

Although technology has improved drastically, it has its own associated issues. Regardless of the rapid progress in technology, companies which adopt fresh technologies undergo challenges (Wen-Lung, 2015). There is the need to investigate the causes of ineffective introduction of IT in organisations. For that matter, researchers give attention to the dynamics influencing effective ushering in of IT (Wen-Lung, 2015). Managers are prepared to be conversant with topical issues surrounding information technology. Also, members of organisations are highly concerned with finding out about the existing subject matters in technology and associated executive matters to better promote IT in business ventures (Porter \&\& Millar, 1985). Strategic issues in MIS attracted many researchers. Unequivocally, major subject matters in MIS caught the attention of a large number of researchers, which led to the emergence of interconnected studies in the 1980s (Wen-Lung, 2015). Among the first to carry out a study on the topic, 'MIS Key Issues', were Ball and Harris (Wen-Lung, 2015). The duo requested 417 members of SIM to rate MIS-connected issues. With reference to the results of the study conducted by Ball and Harris, Dickson et al. carried out a four-round Delphi survey on 52 SIM associates to ascertain the most crucial issues being studied for almost a decade (Wen-Lung, 2015). The study of key issues in MIS continued unabated. Niederman et al., used a three-round Delphi method to survey 241 SIM affiliates. The issues and ratings were made available accompanied by the trend analysis on the issues (Wen-Lung, 2015). Two tendencies were suggested as probable in the 1990s, namely: technology infrastructure-linked issues were more important and efficiency in organizations would receive approval again among business entities (WenLung, 2015).

Development of key issues was renewed through a different approach. Palvia et al., developed key issues in MIS by gathering and digesting articles from seven topmost research journals. A sum of 630 articles, from January 1989 to June 1993, was reviewed with these results: (1) A fresh issue, Known as Expert Systems, was discovered in many articles, though the list of Niederman et al., did not contain it. (2) Executive/Decision Support Systems, Software Development and Telecommunications Systems emerged, hence they were rated the topmost in the list (Wen-Lung, 2015). Research into key MIS issues continued to receive resounding contributions from many academics. Luftman and Kempaiah conducted a study with 112 organisation associates of SIM and called on the participants to grade 38 managerial and 65 technological issues. A related study was carried out and reported by Luftman and Ben-Zvi in a 2010 issue of MIS Quarterly Executive. The researchers engaged 172 members of SIM to rate 39 managerial and 52 technological issues (Wen-Lung, 2015). Review of articles in academic journals broke the monotony of surveys. Although survey was the leading approach to identifying key MIS issues, Palvia et al., made the effort to draw conclusions from reviewing scholarly journals (Wen-Lung, 2015). Analysing and drawing conclusions from diverse academic articles led to the early identification and resolution of pressing issues in MIS. Matters identified in this manner were more research-inclined and showed the likely track of trends, because research journals at times deal with topics prior to their practical application (Wen-Lung, 2015). The identification of relevant issues captured in academic journals gives researchers foreknowledge. Academics and experts become aware of novel topics and draw plans towards the allocation of resources for future investigations (Wen-Lung, 2015). 


\section{A BUSINESS PERSPECTIVE ON INFORMATION SYSTEMS}

Information systems have become an integral part of modern organisations and businesses due to the numerous advantages associated with them. Managers of business firms commit a lot of resources to the establishment and implementation of information systems in expectation of obtaining pecuniary values for their organisations (Asante, Edumadze, Obuobi \& Abbequaye, 2010; Laundon \& Laundon, 2018). Managers always look forward to improving productivity so as to increase the bottom line of their entities through the establishment of information systems. They believe that the returns on commitment of financial resources to the procurement and establishment of information systems will be higher than investment in assets, such as machinery and buildings (Asante et al., 2010). Undoubtedly, the fundamental purpose of building an information system in organisations is to maximise corporate value. The value of an information system is a derivative of the extent to which the system will bring about improved management decisions, more economical business processes and greater organizational profitability (Asante et al., 2010; Brocke, Uebernickel \& Brenner, 2009; Laundon \& Laundon, 2018). Managers adopt information systems other non-pecuniary reasons. Managers build information systems in order to stay in business, beat competition and comply with legal requirements (Asante et al., 2010).

\subsection{DIMENSIONS OF INFORMATION SYSTEMS}

In order to use information systems effectively and reap their benefits, managers must thoroughly understand information systems. In view of the significant economic roles managers expect from the building of information systems, they need to completely comprehend the wider organization, management and information technology dimensions of systems (Asante et al., 2010; Martin, 2017; Laundon \& Laundon, 2018). Information systems are central to organisations. In reality, there would no business for firms such as credit reporting firms without information systems (Asante et al., 2010). Therefore, their introduction in firms changes the status quo of the firms.

The components of the organization, such as people, structure, business processes, policies, politics and culture are affected by the establishment of information systems (Asante et al., 2010; Laundon \& Laundon, 2018). This implies that information systems can create positive or negative changes in people, structure, business processes, policies, politics and culture of the organisation. The introduction of information systems in firms may cause the replacement of some staff, the collapse the organogram, the reengineering of business processes, the modification of existing road maps, the change of organizational leadership and the modus operandi of firms (Martin, 2017).

Management is the hub of every organization. Management's responsibility is to make meaning out of the multiple challenges confronting the organization, make decisions, formulate policies and solve organizational problems (Asante et al., 2010). In view of these functions of managers, they may be likened to sniffing dogs in that they perceive business challenges in the environment. They frame organizational stratagem in response to those challenges and apportion organizational resources for the success of the organization (Asante et al., 2010; Martin, 2017). Managers must add the dimension of leadership to their responsibilities for a better organizational performance. The assumption of leadership responsibilities makes managers both visionary and missionary. The visionary ability of managers makes them dreamers of realties of the world (Asante et al., 2010). This implies that they imagine and design products for the organization to satisfy the needs and wants of consumers. Managers demonstrate their missionary abilities in their quest to manufacture products for satisfying the needs and wants of their clients. Creation of fresh products and services and recreation of the organization intermittently is the hallmark of managers who demonstrate responsible leadership (Asante et al., 2010). Certainly, managers who bear the hallmark of novelty are those who can efficiently dream and create 
products in their organisations. This buttresses the fact that a considerable portion of management's duty is to creative work propelled by novelty and information (Asante et al., 2010; Martin, 2017). Undoubtedly, it is only information technology that can perform rerouting and restructuring the organization.

Change in the way things are done in organisations is a major characteristic of the introduction of information systems. Managers have to cope with this change, and one of the numerous tools managers use to deal with it is information technology (Ackam, Guelu, Guler, Hekim \& Gokhan, 2012). Managers need both computer hardware devices and software programs for processing in an information system. Computer hardware refers to the tangible equipment used to store, process and output data in an information system input, output, storage devices and physical media for connecting these equipment together (Martin, 2017). The computer software is responsible for the coordination of the computer hardware parts in an information system. Telecommunication technology, made up both physical equipment and software, connects the different components and moves data from one physical component to another (Asante et al., 2010). The physical components are usually arranged in a definite pattern to form a network.

\subsection{TYPES OF COMPUTER NETWORKS IN ORGANISATIONS}

Organisational networks can be classified by their jurisdiction. An organizational computer network can be one of types: intranet, extranet. An intranet is a type of network which is limited to the physical boundaries of an organization. An intranet is a network which staff can use to design subject matters, exchange information, work together, get tasks completed and improve the culture of their organization (Eisenhauer, 2019; Asante et al., 2010). An extranet is an organizational intranet which has been extended to stakeholders who operate outside the confines of the organisation. An extranet is similar to an intranet; however, the network is made available to accredited clients, sellers, associates and other interested parties (Eisenhauer, 2019; Asante et al., 2010).

The difference between intranet and extranet is very narrow. It is difficult for many people to comprehend the disparity between intranet and extranet, particularly first time users (Eisenhauer, 2019). Intranets and extranets sustain two dissimilar parts within an organization. In spite of their differences, both intranet and extranet are used to pursue the same objectives: to enhance how workers deal with customers and one another (Eisenhauer, 2019; Asante et al., 2010).

\subsection{BENEFITS OF IMPLEMENTING AN INTRANET}

Organisations have several reasons for which they introduce and implement an intranet. Undoubtedly, there are many justifications for the implementation of an intranet in business organisations (Eisenhauer, 2019; Asante et al., 2010). These justifications may be organization-wide in nature.

Firstly, the presence of intranet in an organization creates and fosters smooth internal communication among employees. Seamless interaction among the workforce of an organization encourages creativeness and efficiency (Eisenhauer, 2019; Asante et al., 2010; Laundon \& Laundon, 2018). There are specific aspects of intranet that rationalise interaction within the whole organisation. Instant messenger, notification and spaces are the features of intranet that simplify communication throughout the whole organization (Eisenhauer, 2019). The seamlessness of communication in an organization makes information ubiquitous in that organization because the use of an intranet breaks departmental barriers.

Secondly, an intranet is an effective organizational project management tool. It engenders a cost-effective project management and workflow systems in firms (Eisenhauer, 2019; Laundon \& Laundon, 2018). An intranet helps in tracking the progress of work among staff and supervisors in firms. Every level of a project is monitored through 
the use of the flexible and interactive features of the intranet Team members of a project interact through the intranet platform to discuss issues concerning the progress of their project through discussion forums, digital workspaces and files (Eisenhauer, 2019; Laundon \& Laundon, 2018).

Thirdly, the intranet is an information hub for the entire organization and its members. The intranet has become the origin of essential knowledge where the entire workforce receive messages or information about the whole organization - make broad enquiries and get access to in-house information (Eisenhauer, 2019; MacAfee \& Brynjolfsson, 2008) The distribution of knowledge in an organization is prerequisite to the success of the entire organization. Through dispersal of knowledge, the talents of employees are developed for the achievement of organizational goals, which benefits the whole company (Eisenhauer, 2019; Laundon \& Laundon, 2018). Also, an intranet creates congruence between the goals of the firm and those of its workers.

\subsection{BENEFITS OF IMPLEMENTING AN EXTRANET}

An organisation establishes an extranet for the benefit of economies of scale. Certainly, firms have their own reasons for the introduction and implementation of an extranet (Asante et al., 2010; Eisenhauer, 2019). These reasons may range from effective employee to the entire organizational performance.

Firstly, firms use extranets to standardise recurrent business processes. With a properlydesigned extranet, firms can place order for inputs or goods through a protected personal network in a computer-generated space (Asante et al., 2010; Eisenhauer, 2019; MacAfee \& Brynjolfsson, 2008). Communication with external stakeholders of an organization occurs instantaneously through extranets. No delays in communication with vendors are experienced because dealings between suppliers and clients happen concurrently, with invoices recorded in addition to other relevant documentations in a central location (Asante et al., 2010; Eisenhauer, 2019; MacAfee \& Brynjolfsson, 2008). This suggests that the use of an extranet economises storage space in computer systems and improves business-tobusiness collaborations.

Secondly, access to an extranet in an organization improves contentment of clients. The continuous availability of extranets in firms renders it beneficial to both an organization and its customers in collaborations (Eisenhauer, 2019; Laundon \& Laundon, 2018; MacAfee \& Brynjolfsson, 2008). Extranets break the physical blockades associated with inflexible work timetable. Customers, at any time, can transfer records, make enquiries or endorse ideas twenty-four-hour-a-day, hence removing the difficulties of work plan (Asante et al., 2010; Eisenhauer, 2019).

Thirdly, security issues are laid to rest when an extranet is introduced in an organization. The installation of a properly-designed extranet in an entity allays the concerns of breach of security (Eisenhauer, 2019). Confidentiality of information is of paramount concern to many organisations globally. An extranet has the ability ensure electronic information protection - emails and other tools regularly used to send documents are secured because of the effective security features of the extranet (Eisenhauer, 2019).

\section{THE FOUR MAJOR TYPES OF INFORMATION SYSTEMS}

Organisations adopt information systems based on organisational levels. Primarily, there are four main information systems established in organisations, namely: transactional processing systems (TPS) for operational level, management information systems (MIS) and decision support systems (DSS) for management level and executive support systems (ESS) for executive level (Asante et al., 2010; Eisenhauer, 2019; Laundon \& Laundon, 2018). Each of these types of information systems has its own specialized functions in tandem with the main functional areas of a typical organization. The conventional information systems adopted in organisations are created to support employees or 
executives at each echelon and in the functional areas of human resources, accounting and finance, manufacturing and production and marketing and sales (Asante et al., 2010; Eisenhauer, 2019; Laundon \& Laundon, 2018).

\subsection{TRANSACTION PROCESSING SYSTEMS (TPS)}

Transaction processing systems are the fundamental business systems in an organization. They serve the operational echelon of the entity (Asante et al., 2010). Transactional processing systems can be likened to the book of first entry, sales journal. They are computerized systems execute and document regular dealings, such as sales order entry, payroll, employee record keeping, hotel reservation and delivery, central to the conduct of business (Asante et al., 2010; Laundon \& Laundon, 2018). Business activities carried out at the operational level are predetermined and well-thought-out in nature. Tasks, resources and goals of managers at this level of management are pre-decided and follow set standards - the decision to grant an amount of loan to a client (Asante et al., 2010).

Transaction processing systems are the lifeblood of business organisations. They are pivotal to the survival of the organization in that their malfunction for a few hours can result in its shutdown (Asante et al., 2010; Laundon \& Laundon, 2018). TPS is an internal monitoring tool for managers. Managers use it to supervise in-house operations and the entity's rapport with the outside environment (Laundon \& Laundon, 2018). The centrality of TPS to the existence and survival of organizations makes it the bedrock of all other information systems in an organization.

\subsection{MANAGEMENT INFORMATION SYSTEMS (MIS)}

Management information system (MIS) is basically the study of information systems in organisations. The terminology is also used to refer to a class of information system which serves management-level purposes (Asante et al., 2010; Laundon \& Laundon, 2018). Management information system (MIS) helps managers to execute their functions of planning, controlling and decision making properly. MIS assists mid-level managers to generate reports on a firm's present and past internal performance (Asante et al., 2010). This implies that MIS is equipped with tools which summarise and report on the fundamental business activities of the firm. Management information systems are essentially concerned with the generation of weekly, monthly and yearly reports for the perusal of managers in decision making (Asante et al., 2010; Laundon \& Laundon, 2018). MIS is generally inflexible in that it produces answers for frequently-asked questions which have been itemized in advance and have a fixed procedure in providing responses to the. Management information systems are mostly structured and incapable of conducting critical analysis on transactions (Ackam et. al., 2012; Asante et al., 2010; Laundon \& Laundon, 2018). They are purposely designed to summarise and compare transactions of different timelines.

\subsection{DECISION SUPPORT SYSTEMS (DSS)}

Decision support systems are designed to be engaged at management level of an organization. They aid managers to make decisions that are exceptional, fluid and highly unpredictable (Asante et al., 2010). DSS often utilize in-house information in addition to information from the industry. The internal information from both TPS and MIS of a firm and the external information, in the form of prices of shares and products of competitors are analysed in the DSS (Asante et al., 2010; Laundon \& Laundon, 2018). DSS is used by managers to conduct simulations on decisions.

DSS is analytically more powerful than any other information system, contains numerous models that examine data and compresses huge quantities of data into an easyto-analyse form for decision makers (Laundon \& Laundon, 2018). These information systems contain user-friendly and interactive software. The user of these systems can alter 
hypotheses, include fresh concepts and ask 'what if' questions (Asante et al., 2010; Laundon \& Laundon, 2018).

\subsection{EXECUTIVE SUPPORT SYSTEMS (ESS)}

Executive support systems are designed for senior managers of an organization. They support the strategic level of a company to make decisions (MacAfee \& Brynjolfsson, 2008). They are highly flexible in nature. ESS deals with decisions which warrant subjectivity, assessment and discernment in that there is no predetermined formula for obtaining as answer (Asante et al., 2010). ESS draws information from both inside and outside of the organization. They have been created to absorb information concerning outside-of-orgnisation issues, to be precise, tax regulations and business competitors; however, they pull condensed information from MIS and DSS (Asante et al., 2010; Laundon \& Laundon, 2018).

Executive support systems are indispensable in the management of modern organisations. They sieve, condense and trail essential information, showing the information of highest relevance to top executives (Asante et al., 2010; Laundon \& Laundon, 2018). They perform these functions because they are equipped with sophisticated illustrative software, hence present information in graphic forms from numerous sources. Top managers get access to the information via a web-interfaced portal which forwards combined individualized business subject matters from different sources (Asante et al., 2010; Laundon \& Laundon, 2018). Executive support systems are not problem-specific analytical in nature; they are rather general in character. They deliver comprehensive computing and communications facility that can be relevant to a varying collection of glitches (Asante et al., 2010; Laundon \& Laundon, 2018).

ESS provides responses to universal questions. Questions such as: In which business must we be? What are the strengths of our rivals? What fresh acquisitions would safeguard our firm against recurring business blows? Which amount of products shall dispose of to fund a new acquisition? (Asante et al., 2010). ESS interacts with other systems from both inside and outside of an organization. It comprises computer terminals which possess highly interactive graphics and collaborative features that are able to retrieve past and feasible data from in-house organizational systems and outside data banks such as Standard and Poor and Dow Jones News (Asante et al., 2010). Executive support systems are highly user-friendly and have easy-to-use graphic interfaces. They are created for the use of top managers who are not well-vexed in computer-related information systems (Asante $e t$ al., 2010; Laundon \& Laundon, 2018).

\section{CONCLUSION}

The advent of information technology has turned every aspect of human life round. Information technology has assumed an inseparable dimension in the lives of individuals, organisations and society in general in that it provides many socio-economic benefits for its users. The introduction of information technology in organisations for easy management of organizational information has suffered numerous challenges but researchers and technologists are incessantly toiling to create congruence between information technology and information management in organisations. These researchers and technologists are bent on integrating information technology into business organisations due to the financial payoffs and competitive advantages identified to be associated with the introduction of information technology in the management of organizational information.

The analysis of the impact of information systems on the organization's structure, its management and existing technology is an essential dimension to be considered in the introduction of any information system into organisations. Modern information systems require computer networks - intranets or extranets, depending on the intentions of top management in the establishment of the information system. Networked information 
systems provide numerous benefits for organisations. Seamless sharing of information, effective management of projects, centralization of information, streamlining of business processes, improved customer satisfaction and improved information security are the benefits of using computer-networked information systems in firms. To fully enjoy these benefits, organisations are required to invest in the four major types of information systems - Transaction Processing Systems (TPS) for lower level managers, Management Information Systems (MIS) and Decision Support Systems (DSS) for middle level managers and Executive Support Systems (ESS) for top executives.

\section{REFERENCES}

[1] Asante, A., Edumadze, J. K. E. Obuobi, D. \& Abbequaye, G. (2010).Management information systems II. Ghana, Accra: Mercury Press.

[2] Ball, L. D. \& Harris, R. G. H. (1982). SMIS members: A membership analysis. MIS Quarterly, 6(1), 1938.

[3] Dickson, G. W. Leitheiser, R. L., Wetherbe, J. C. \& Nechis, M. (1984). Key information systems issues for the 1980s. MIS Quarterly, 8(3), 135-159.

[4] Eisenhauer, T. (2019). Intranets vs extranets - What is the difference? Retrieved from: www.axerosolutions.com

[5] Laundon, K. C. \& Laundon, J. P. (2018). Management information systems. (15 th ed.). New York, NY:Pearson Education Inc.

[6] Luftman, J. \& Kempaiah, R. (2008). Key issues for IT executives 2007. MIS Quarterly Executive, 7(2), 99-112.

[7] MacAfee, A. \& Brynjolfssoin, E. (2008). Investing in the IT that makes a competitive difference. Harvard Business Review (July/August 2008).

[8] Martin, (2017). Management information systems (MIS): Definition and how it works. Retrieved from: www.cleverism.com

[9] Niederman, F. \& Brancheu, J. C. \& Wetherbe, J. C. (1991). Information systems management issues for the 1990s. MIS Quarterly, 15(4), 475-500.

[10] Obuobi, D., Edumadze, J. K. E., Asante, A. \& Abbequaye, G. (2004). Management information systems I. Ghana, Cape Coast: University Press.

[11] Palvia, P. C., Rajagopalan, B., Kumar, A. \& Kumar, N. (1996). Key information systems issues: An analysis of MIS publications. Information Processing and Management, 32(3), 345-355.

[12] Porter, M. E. \& Millar, V. E. (1985). How information gives you competitive advantage. Harvard Business Review, 63(4), 149-160.

[13] Wen-Lung, S. (2015). Management information systems issues: Co-citation analysis of journal articles. International Journal of Electronic Commerce Studies, 6(1), 145-162. 